\title{
Microfold (M) cells: important immunosurveillance posts in the intestinal epithelium
}

\author{
NA Mabbott ${ }^{1}$, DS Donaldson ${ }^{1}, \mathrm{H} \mathrm{Ohno}^{2}$, IR Williams ${ }^{3}$ and A Mahajan ${ }^{1}$
}

The transcytosis of antigens across the gut epithelium by microfold cells ( $M$ cells) is important for the induction of efficient immune responses to some mucosal antigens in Peyer's patches. Recently, substantial progress has been made in our understanding of the factors that influence the development and function of $\mathrm{M}$ cells. This review highlights these important advances, with particular emphasis on: the host genes which control the functional maturation of $\mathrm{M}$ cells; how this knowledge has led to the rapid advance in our understanding of $\mathrm{M}$-cell biology in the steady state and during aging; molecules expressed on $\mathrm{M}$ cells which appear to be used as "immunosurveillance" receptors to sample pathogenic microorganisms in the gut; how certain pathogens appear to exploit $M$ cells to infect the host; and finally how this knowledge has been used to specifically target antigens to $M$ cells to attempt to improve the efficacy of mucosal vaccines.

\section{INTRODUCTION}

A single layer of epithelial cells provides a protective barrier against the substantial bacterial burden within the intestinal lumen. However, the epithelia overlying the organized lymphoid follicles of the gut-associated lymphoid tissues (GALT), including the Peyer's patches, their equivalents in the cecum and colon, and isolated lymphoid follicles, are specialized for sampling the lumenal contents. Under steadstate conditions, approximately $10 \%$ of the epithelial cells within these follicle-associated epithelia (FAE) are microfold (M) cells. ${ }^{1-3}$ These cells have unique morphological features, including the presence of a reduced glycocalyx, irregular brush border and reduced microvilli. Furthermore, in contrast to the neighboring enterocytes within the FAE, M cells are highly specialized for the phagocytosis and transcytosis of gut lumen macromolecules, particulate antigens and pathogenic or commensal microorganisms across epithelium (Figure 1). Following their transcytosis across the FAE, antigens exit into the intraepithelial pocket beneath the M-cell basolateral membrane, which contains various populations of lymphocytes and mononuclear phagocytes (MNP, a heterogenous population of macrophages and classical dendritic cells ${ }^{4-6}$ ). This specialized microenvironment beneath the $\mathrm{M}$-cell enables the efficient transfer of lumenal antigens to MNP. ${ }^{7,8}$ Studies show that in the absence of M cells, or antigen sampling by $\mathrm{M}$ cells, antigen-specific T-cell responses in the Peyer's patches of mice orally infected with Salmonella Typhimurium are reduced. ${ }^{2,9}$ Thus, the efficient M-cellmediated sampling of gut lumenal antigens is considered an important initial step in the induction of some mucosal immune responses. ${ }^{2,7-9}$

In this review, we mostly describe the recent progress that has been made in our understanding of the immunobiology of $M$ cells in the intestine, but it is important to note that M cells are not only restricted in the FAE overlying the GALT. For example, cells with typical features of $M$ cells have been reported in the murine nasal passage epithelium, identifying a novel, NALT (nasal-associated lymphoid tissues)-independent mode of antigen sampling in the respiratory tract. ${ }^{10}$ In the intestine, $\mathrm{M}$ cells have also been described within the villous epithelium, ${ }^{8,11,12}$ but whether these cells are functionally equivalent to those within the FAE overlying the organized lymphoid follicles remains to be determined. ${ }^{13}$

\section{CELLS ARISE AND DIFFERENTIATE FROM LGR5 ${ }^{+}$STEM CELLS IN THE CRYPTS IN A RANKL- AND SPI-B-DEPENDENT MANNER}

As $\mathrm{M}$ cells are considered to have an important role in the induction of specific mucosal immune responses in the Peyer's patches, ${ }^{2,9}$ their manipulation may improve the efficacy of

\footnotetext{
${ }^{1}$ The Roslin Institute and Royal (Dick) School of Veterinary Sciences, University of Edinburgh, Easter Bush, Midlothian, Scotland, UK. ${ }^{2}$ RIKEN Center for Integrative Medical Sciences (IMS-RCAI), Tsurumi, Yokohama, Japan and ${ }^{3}$ Department of Pathology, Emory University School of Medicine, Atlanta, Georgia, USA. Correspondence: NA Mabbott (neil.mabbott@roslin.ed.ac.uk)
} 

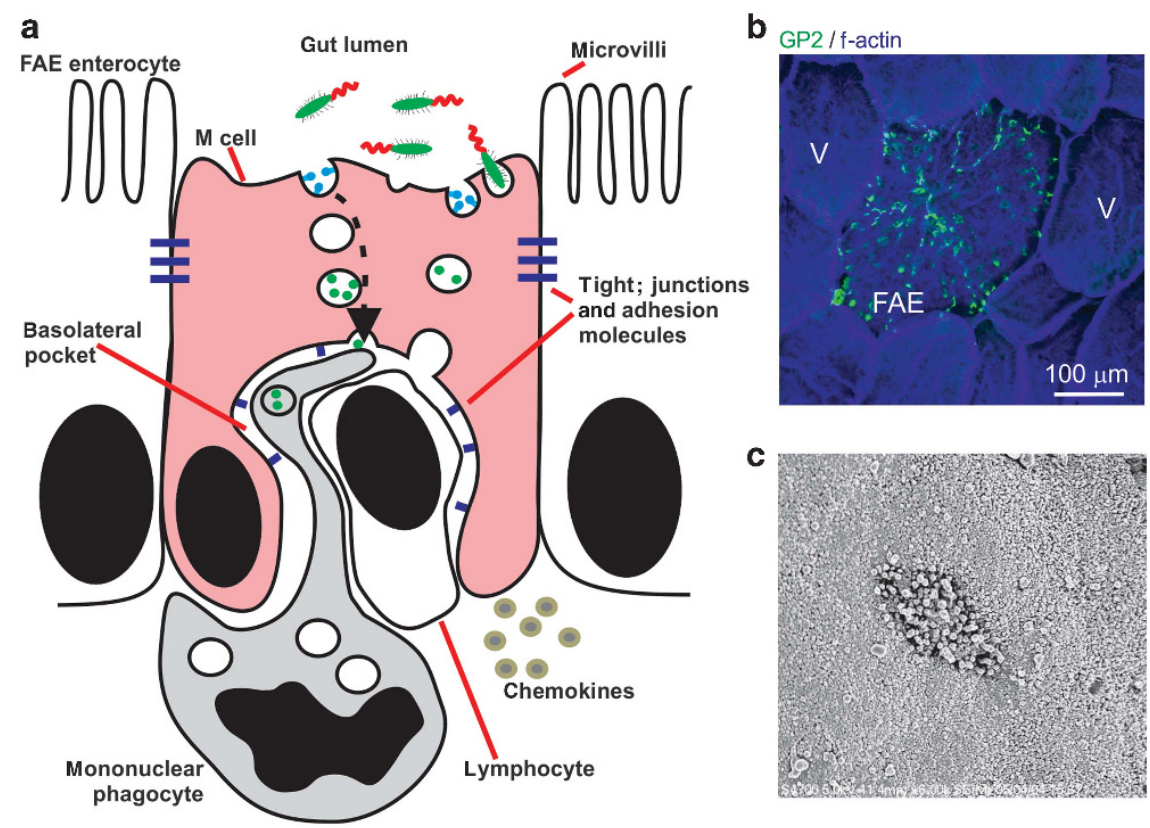

Figure 1 The morphological features of M cells. (a) Cartoon illustrating the morphological features of M cells. Note the lack of microvilli and basolateral pocket containing a mononuclear phagocyte and a lymphocyte. (b) Whole-mount immunohistochemical analysis of glycoprotein 2 (GP2) ${ }^{+}$mature M cells (green) in the follicle-associated epithelia (FAE) of a mouse Peyer's patch. Note the regular distribution of the M cells across the FAE. Note also that in the steady-state $\mathrm{M}$ cells are restricted to the FAE and are mostly absent from the surrounding villi. Tissue counterstained to detect f-actin (blue). V, villi. (c) Scanning electron micrograph of the apical surface of a M-cell. Note characteristic lack of/blunted microvilli.

mucosal vaccines or help develop strategies to block transmission of some orally acquired infections (discussed below). Many studies have therefore attempted to elucidate the molecular mechanisms which regulate M-cell differentiation in the gut epithelium. In the intestine, almost all epithelial cell lineages develop from intestinal epithelial stem cells within the crypts. The dome-associated crypts surrounding the FAE, and the villous crypts at the base of the villi, each contain cycling leucine-rich repeat-containing $G$ protein-coupled receptor(Lgr5-) positive stem cells intermingled among Paneth cells. ${ }^{14}$ These stem cells produce highly proliferative daughter cells, which upon appropriate stimulation can differentiate into all the epithelial cell populations within the small intestine, including the enterocytes, goblet cells, enteroendocrine cells, tuft cells, and Paneth cells. Lineage-tracing studies using transgenic mice expressing a reporter gene (LacZ) under control of the Lgr5 promoter have confirmed that all the epithelial cells within the FAE, including $\mathrm{M}$ cells, are derived from the cycling Lgr5 ${ }^{+}$stem cells within the dome-associated crypts $^{15-17}$ (Figure 2).

In the mouse intestine under steady-state conditions, $\mathrm{M}$ cells are mostly confined to the FAE overlying the organized lymphoid follicles of the GALT. As both the dome-associated crypts and the villous crypts throughout the intestine contain cycling Lgr $5^{+}$stem cells, additional signals from the stromal and immune cells beneath the FAE in the subepithelial dome (SED) are required to induce $\mathrm{M}$-cell differentiation, and in doing so restrict these cells to the epithelia overlying organized lymphoid follicles. The tumor necrosis factor (TNF) superfamily member receptor activator of nuclear factor- $\kappa \mathrm{B}$ ligand
(RANKL) signals via its receptor RANK (receptor activator of nuclear factor $-\kappa \mathrm{B})$, which is expressed by epithelial cells throughout the intestine. However, in the intestine RANKL is selectively expressed by the subepithelial stromal cells beneath the FAE. ${ }^{18,19}$ Analysis of RANKL-deficient mice, and administration of exogenous RANKL in vivo, show this cytokine is a critical factor that controls the differentiation of RANKexpressing enterocytes into $\mathrm{M}$ cells. ${ }^{20}$ Under steady-state conditions, the only RANK-expressing intestinal epithelial cells that are exposed to sufficient RANKL from any source to respond in a detectable way are those in the FAE that are immediately above the RANKL-expressing subepithelial stromal cells. These data imply that in the context of M-cell differentiation, much of the RANKL-RANK signalling is delivered by the intact transmembrane cytokine following direct cell contact, rather than systemic dissemination of soluble RANKL. However, the possibility cannot be entirely excluded that both the membrane and soluble forms of RANKL are expressed by SED stromal cells. Accordingly, in the steady state, villous enterocytes situated distant from organized lymphoid structures are not exposed to enough RANKL to show any induction of the genes that regulate M-cell differentiation. ${ }^{2,20}$ Thus, the restriction of RANKL expression to the subepithelial stromal cells ensures that the differentiation of $\mathrm{Lgr}^{+}$stem cells into $\mathrm{M}$ cells is spatially restricted to the FAE overlying GALT (Figure 2). Consistent with this theory, the systemic treatment of mice with RANKL induces the ectopic differentiation of enterocytes into $\mathrm{M}$ cells in the villous epithelium., ${ }^{2,20}$ To help to focus the effects of soluble RANKL to the overlying FAE, it is plausible that the 

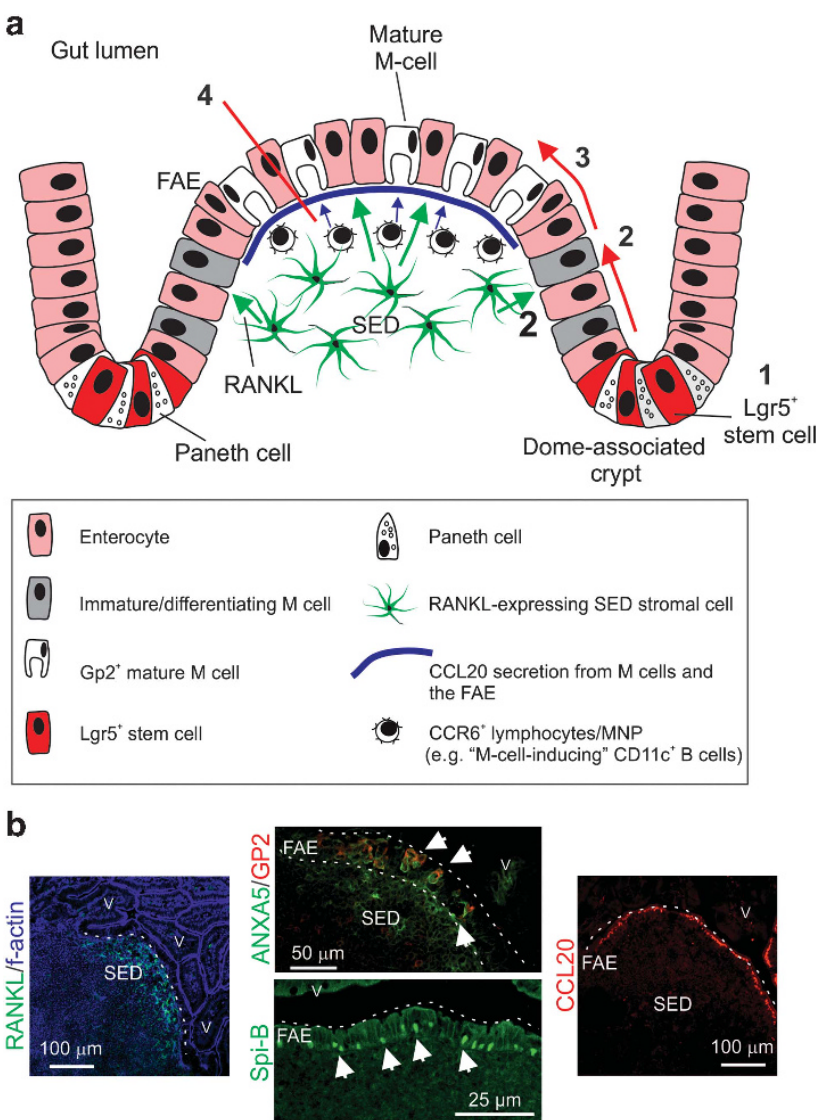

Figure $2 \mathrm{M}$ cells differentiate from $\mathrm{Lgr}^{+}$stem cells in the crypts in a receptor activator of nuclear factor- $\kappa B$ ligand (RANKL)- and Spi-Bdependent manner. (a, 1) All epithelial cell lineages, including $M$ cells, develop from Lgr5 $^{+}$intestinal epithelial stem cells within the crypts. ${ }^{15}$ $(\mathbf{a}, 2)$ In the intestine, RANKL is selectively expressed by the subepithelial stromal cells beneath the follicle-associated epithelia (FAE). ${ }^{18,19}$ RANKL controls the differentiation of $\mathrm{Lgr}^{+}$stem cell-derived RANK-expressing enterocytes into M cells. ${ }^{15,20}(\mathbf{a}, 3)$ RANKL also induces the expression of the ETS (E26 transformation-specific) family transcription factor Spi-B. ${ }^{2}$ The subsequent differentiation of the annexin A5 (ANXA5) ${ }^{+}$, MARCKS $^{-}$ (myristoylated alanine-rich $\mathrm{C}$-kinase substrate)-like protein $1^{+}$immature $\mathrm{M}$ cells into functionally mature glycoprotein $2(\mathrm{GP} 2)^{+}$, tumor necrosis

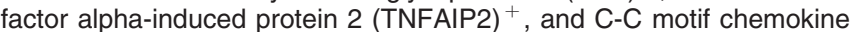
ligand 9 (CCL9) ${ }^{+} \mathrm{M}$ cells is regulated by intrinsic expression of Spi-B..$^{2,15,22}(\mathbf{a}, \mathbf{4})$ The chemokine CCL20 is specifically expressed by the $\mathrm{FAE},{ }^{34}$ and in the Peyer's patches, it mediates the chemoattraction of C-C motif chemokine receptor 6 (CCR6)-expressing lymphocytes and leukocytes towards the FAE. ${ }^{35}$ When CCL20-CCR6 signalling is impeded, $\mathrm{M}$-cell maturation is likewise impeded. ${ }^{36-38}$ Data suggest that a unique subset of $\mathrm{CCR}^{\text {hi }}{ }^{\mathrm{C}} \mathrm{CD11} \mathrm{c}^{\text {int }} \mathrm{B}$ cells specifically migrates towards FAEderived CCL20 and promotes M-cell differentiation. ${ }^{37}$ However, as M-cell maturation can be induced in an in vitro "gut organoid" model system containing only epithelial cell elements, ${ }^{15}$ it is plausible that these CCL20-CCR6-mediated lympho-epithelial interactions most likely provide accessory factors that enhance M-cell differentiation. (b) Immunohistochemical analysis of the distribution of RANKL (green, left-hand panel), ANXA5, GP2 (green and red, respectively, upper-middle panel), Spi-B (green, lower-middle panel), and CCL20 (red, right-hand panel) in mouse Peyer's patches. Dotted lines indicate the boundary of the FAE. Arrows indicate positively immunostained $M$ cells. V, villi. MNP, mononuclear phagocytes; SED, subepithelial dome.

range of its activity is further restricted by binding to osteoprotegerin, a secreted decoy receptor of RANKL. ${ }^{21}$

Distinct transcription factors control the expression of the sets of genes that ultimately determine the differentiation of crypt stem cell-derived progenitor cells into terminally differentiated intestinal epithelial cell populations. The same is true for $\mathrm{M}$ cells. The RANKL-induced, M-cell intrinsic expression of the ETS (E26 transformation-specific) transcription factor Spi-B specifically regulates the maturation of immature $\mathrm{M}$ cells into functionally mature $\mathrm{M}$ cells. Although immature M-cell differentiation appeared to be unaffected in Spi-B-deficient $\left(\right.$ Spi- $\left.\mathrm{B}^{-1-}\right)$ mice, their FAE were are almost completely deficient in functionally mature $\mathrm{M}$ cells. ${ }^{2,15,22}$ Thus, the terminal differentiation of $\mathrm{M}$ cells, as for other intestinal epithelial cell lineages, is regulated by a specific transcription factor (Figure 2).

Transcriptomic profiling studies have helped to identify those genes which are expressed by functionally mature $\mathrm{M}$ cells and regulated by Spi-B, including: glycoprotein 2 (Gp2), TNF alpha-induced protein 2 (Tnfaip2), and C-C motif chemokine ligand 9 (Ccl9) (discussed below). Others such as annexin A5 (Anxa5) and Marcksl1 (which encode for annexin A5 and MARCKS (myristoylated alanine-rich C-kinase substrate)-like protein 1, respectively) are expressed by immature (differentiating) $\mathrm{M}$ cells in response to RANKL stimulation but are regulated independently of Spi-B. ${ }^{2,13,15,23-26}$ Although their precise roles are uncertain, the immature $\mathrm{M}$-cell markers such as ANXA5 and MARCKS-like protein 1 may be involved in cytoskeletal regulation to help adapt the differentiating epithelial cell towards one specialized for transcytosis. ${ }^{27,28}$

An independent study has also investigated the requirement for Spi-B in M-cell differentiation. ${ }^{22}$ In this study, two distinct monoclonal Abs were used to detect $\mathrm{M}$ cells: one $\mathrm{mAb}$ specific for GP2 (discussed below); and the other mAb NKM 16-2-4, which specifically recognizes sites of $\alpha-1,2$ fucosylation ${ }^{25,29}$ and has a greater degree of specificity for M cells than the lectin Ulex europaeus agglutinin I (UEA-1, which also binds $\alpha-1,2$ fucose with high affinity). Although the expression of the mature $\mathrm{GP}^{+} \mathrm{M}$ cells was completely absent in the FAE of Spi-B ${ }^{-1-}$ mice, a small number of mAb NKM 16-2-4 ${ }^{+} \mathrm{M}$ cells were evident $\left(\sim 10 \mathrm{mAb}\right.$ NKM $16-2-4^{+} \mathrm{M}$ cells/FAE) ${ }^{22}$ One interpretation of these apparently contradictory data is that a small population of $\mathrm{M}$ cells in the FAE may mature independently of Spi-B and, as a consequence, do not express proteins such as GP2 on their surfaces. ${ }^{22}$ Alternatively, it is plausible that the remaining $\mathrm{mAb}$ NKM $16-2-4^{+}$cells in the FAE of Spi-B ${ }^{-1-}$ mice were not fully differentiated $\mathrm{M}$ cells or represented other populations of fucosylated enterocytes.

$\mathrm{M}$ cells appear to be regularly spaced in the FAE implying that their distribution is highly regulated (Figure 1b). Indeed, while all the epithelial cells in the FAE derive from $\mathrm{Lgr} 5^{+}$stem cells ${ }^{15}$ and are exposed to high levels of RANKL stimulation from the stromal cells in the SED, ${ }^{20}$ only a proportion of the cells are destined to become $\mathrm{M}$ cells. Hsieh et al. ${ }^{30}$ have questioned how this distribution may be regulated. Notch receptors and their ligands have important roles in determining cell fate and tissue patterning. For example, direct cell-cell signalling, where the expression of a Notch ligand by one cell may influence the fate of an adjacent cell expressing a Notch receptor. Consistent with this role, Notch and the ligand Jagged 1 also appear to influence the patterning of $M$ cells across 

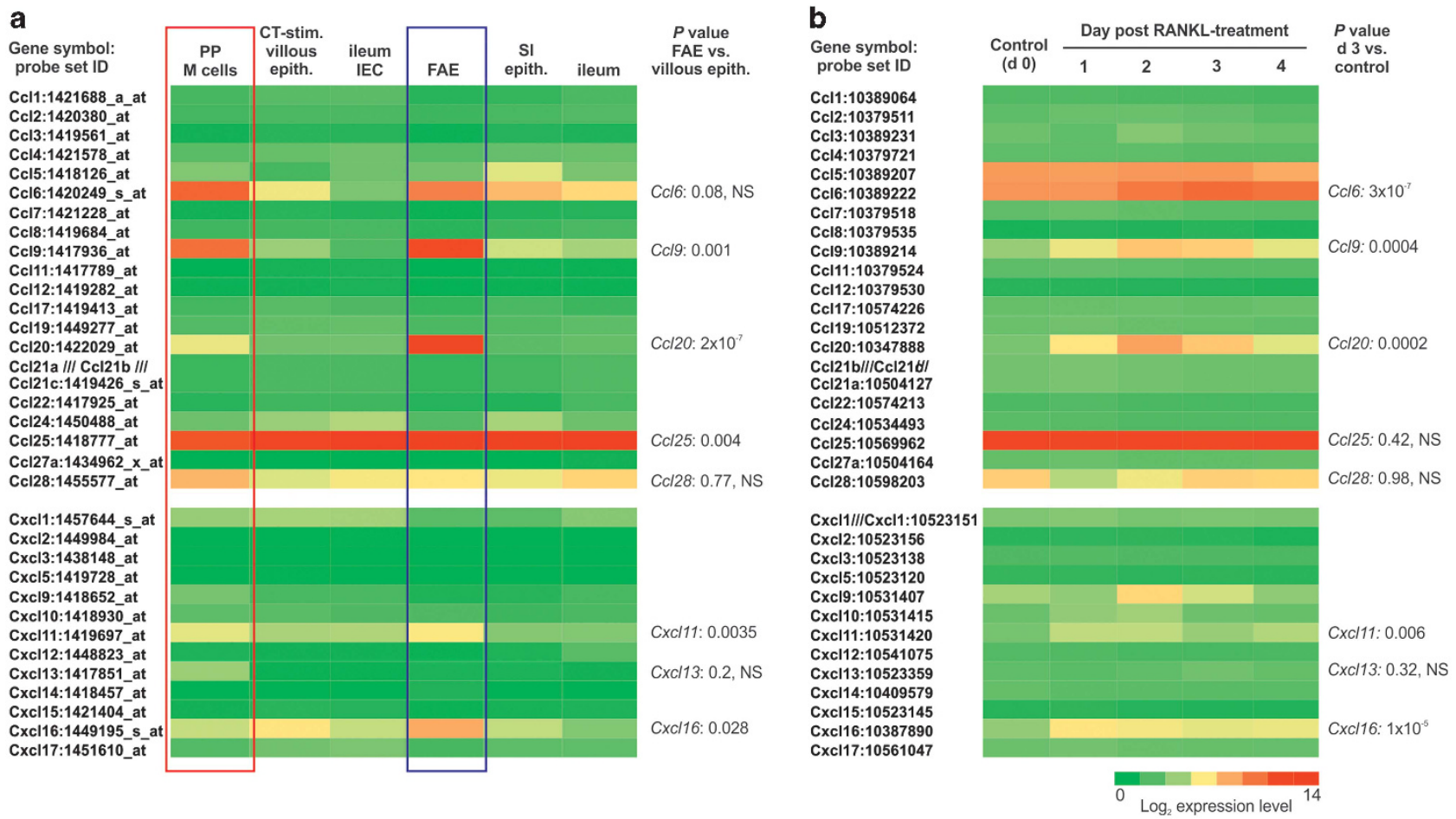

Figure 3 Retrospective comparison of chemokine gene expression by $M$ cells and the follicle-associated epithelia (FAE). (a) Heat map showing the mean expression of profile of multiple chemokine-encoding probe sets in the samples of Peyer's patch (PP) M cells, cholera-toxin-induced (CT-stim.) villous $\mathrm{M}$ cells and ileum intestinal epithelial cells (GSE7838), ${ }^{25} \mathrm{FAE}, \mathrm{M}$ cells, ${ }^{26}$ and ileum (GSE13908). ${ }^{68}$ These data were performed on Affymetrix MOE430_2 mouse genome expression arrays (Affymetrix, Santa Clara, CA). Each column represents the mean probe set intensity (log ${ }_{2}$ ) for all the samples from each source $(n=2-4)$. Significant differences between groups were sought by analysis of variance. $P$-values for those genes which were expressed significantly $(P<0.05)$ within the FAE and by $\mathrm{M}$ cells at levels $>$ two fold when compared with the villous epithelium are indicated. (b) Effect of receptor activator of nuclear factor- $\kappa B$ ligand (RANKL)-stimulation on the expression of chemokine-encoding genes in the villous epithelium. These data (GSE37861) $^{2}$ were performed on Affymetrix mouse gene 1.0 ST expression arrays (equivalent chemokine-related probe sets are shown). Each column represents the mean probe set intensity $\left(\log _{2}\right)$ for all the samples from each source $(n=3)$. $P$-values for those genes that were significantly upregulated $>$ two fold at day 3 after RANKL-treatments when compared with controls (day 0 ) are indicated. Representative probe set are shown when multiple probe sets for a gene were present on the arrays. NS, not significant.

the FAE. ${ }^{30}$ The optimal distribution of $\mathrm{M}$ cells across the FAE may be important to ensure efficient immune surveillance, while at the same time maintaining the integrity of the FAE.

Analysis of cleaved caspase-3 expression in the intestinal epithelia of rats suggested that almost all the epithelial cells within the villi appear to be undergoing apoptosis, whereas the onset of apoptosis within the FAE has been reported to be delayed. ${ }^{31}$ The differentiation and maturation of $\mathrm{M}$ cells from Lgr5 $^{+}$stem cells within the crypts takes $\sim 3-4$ days. ${ }^{2,20}$ Whether this apparent delay to the progression of apoptosis in the FAE enables $\mathrm{M}$-cell differentiation to be sustained for longer periods is uncertain.

\section{LYMPHO-EPITHELIAL FACTORS INFLUENCE M-CELL DIFFERENTIATION IN THE FAE}

In addition to the important stimuli provided to the differentiating M cells in the FAE by SED stromal cells, lympho-epithelial cell interactions also influence their development. Indeed, a role for hematopoietic cells such as B cells in inducing M-cell differentiation is well established. ${ }^{32,33}$ In the small intestine, the chemokine CCL20 is specifically expressed by the FAE, ${ }^{34}$ and in the Peyer's patches, it mediates the chemoattraction of C-C motif chemokine receptor 6 (CCR6)-expressing lymphocytes and leukocytes towards the FAE. ${ }^{35}$ Mice deficient in CCR6 $\left(\mathrm{CCR6}^{-1-}\right.$ mice), the sole receptor for CCL20, have a significantly reduced frequency of mature $\mathrm{GP} 2^{+} \mathrm{M}$ cells in the $\mathrm{FAE}^{36-38}$ implying that the impaired migration of CCR6expressing lymphocytes or leukocytes towards the FAE impedes M-cell differentiation due to reduced lympho-epithelial cell interactions. Alternatively, this may also be due to an altered cytokine milieu in the SED. In the Peyer's patches, a unique subset of CCR $6{ }^{\text {hi }} \mathrm{CD} 11 \mathrm{c}^{\mathrm{int}} \mathrm{B}$ cells has been reported to specifically migrate towards FAE-derived CCL20 and promote M-cell differentiation ${ }^{37}$ (Figure 2). In the absence of CCR6, these cells could not migrate towards the FAE and M-cell differentiation was impeded, whereas their transfer into CCR6 ${ }^{-1-}$ mice coincided with restored M-cell maturation. Further analysis shows that although mature $\mathrm{M}$ cells are dramatically reduced in the FAE of $\mathrm{CCR6}^{-1-}$ mice, immature ANXA5-expressing $\mathrm{M}$ cells are retained. ${ }^{36-38}$ These data imply that CCL20-CCR6signaling blockade in CCR6 ${ }^{-1-}$ mice does not impact on the initial induction of M-cell differentiation but specifically impedes their functional maturation. Enteroinvasive bacteria, including $S$. Typhimurium and the pathogenic bacteria $S$. Enteritidis and Listeria monocytogenes, ${ }^{39}$ have each been shown to induce CCL20 expression by intestinal epithelial cells. This 
pathogen-mediated stimulation of CCL20 expression may be part of the host's response to infection to attempt to enhance the "immunosurveillance" of the gut lumen. Alternatively, these pathogens may exploit this to potentially aid their uptake from the gut lumen by stimulating the differentiation of enterocytes into $\mathrm{M}$ cells.

The factors provided by B cells (or other hematopoietic cell populations) that stimulate $\mathrm{M}$-cell differentiation are uncertain but may involve signalling between $\mathrm{B}$ cells and the M-cell progenitor cells via $\mathrm{CD} 137 \mathrm{~L} / \mathrm{CD} 137^{8,40}$ or macrophage migration inhibitory factor. ${ }^{41}$ The expression of CCL20 in the intestinal epithelium is rapidly induced by RANKL stimulation (Figure 3) and enhanced by TNFR and lymphotoxin $\beta$ receptor stimulation. $^{2,42-44}$ However, hematopoietic cell populations within the Peyer's patches, including the $\mathrm{CD} 11 \mathrm{c}^{+} \mathrm{B}$ cells, do not express RANKL, ${ }^{37}$ and B-cell-derived TNF and lymphotoxin are each dispensable for the development of FAE and M cells. ${ }^{45}$ Although the above studies together provide a plausible model by which lympho-epithelial interactions may contribute to the functional maturation of $\mathrm{M}$ cells, it is important to note that M-cell maturation can be induced in an in vitro "gut organoid" model system, which contains only epithelial cell elements. ${ }^{15}$ Although the in vivo environment may differ significantly from that of an in vitro gut organoid, these data suggest that these lympho-epithelial interactions most likely provide accessory factors that enhance M-cell differentiation (Figure 2).

\section{CELLS ARE EQUIPPED WITH AN ARRAY OF MOLECULES TO SAMPLE LUMENAL ANTIGENS}

$M$ cells provide an efficient portal through which gut lumenal antigens can be delivered to the underlying mucosal lymphoid tissues. In order to achieve this, $\mathrm{M}$ cells, in contrast to enterocytes, have a high endocytic and transcytotic capacity. Whether the sampling and transcytosis of microoganisms/ antigens by $\mathrm{M}$ cells occurs predominantly in a non-selective manner as a consequence of constitutive "bulk sampling" of the lumenal contents, specifically via receptor mediated endocytosis, or both is uncertain. However, data from many studies suggest that M cells express a variety of "immunosurveillance" receptors on their apical surfaces that enable them to sample a diverse range of microbial pathogens and antigens.

\section{Glycoprotein 2}

Independent sets of gene-profiling experiments aimed at identifying molecules that were expressed highly by $\mathrm{M}$ cells, but not by other enterocyte populations, identified GP2 as a specific M-cell marker. ${ }^{24,25}$ This glycosylphosphatidylinositolanchored protein is highly expressed on the apical surface of $M$ cells and selectively binds to the FimH component of the type I pili on the outer membrane of certain pathogenic and commensal bacteria, such as Eschericia coli and S. Typhimurium. In the absence of GP2 expression by $M$ cells, the transcytosis of type-I-piliated bacteria was impeded. ${ }^{9}$ Furthermore, GP2-dependent bacterial transcytosis by $\mathrm{M}$ cells was shown to be important for the immunosurveillance of FimH ${ }^{+}$ bacteria in the gut lumen. ${ }^{9}$

\section{Uromodulin}

A homolog of GP2, uromodulin (UMOD or Tamm-Horsfall protein), has also been reported to be specifically expressed by M cells. ${ }^{15,22}$ Secreted UMOD is a urinary protein that can also bind specifically to type-I-piliated E. coli and may function to prevent these bacteria from binding to uroepithelial cells. ${ }^{46}$ Although the expression of Umod by Peyer's patch M cells is much lower than $G p 2$, their similar characteristics imply that each protein may aid the sampling of $\mathrm{FimH}^{+}$bacteria in the gut lumen. ${ }^{9,22}$

\section{Cellular prion protein}

Continuing with the immunosurveillance theme, the cellular prion protein $\left(\mathrm{PrP}^{\mathrm{C}}\right)$ is also expressed highly on the apical membrane of $\mathrm{M}$ cells ${ }^{26}$ and has been implicated in the sampling of microorganisms from the gut lumen. ${ }^{47}$ The GPI-anchored $\operatorname{PrP}^{\mathrm{C}}$ protein is expressed highly by many cell populations in mammals, but its precise function remains uncertain as PrPdeficient mice do not show an obvious phenotype. The Gramnegative bacterium Brucella abortus is a facultative intracellular pathogen and can express the heat-shock protein 60 (Hsp60) on its cell surface. Data show that $\operatorname{PrP}^{\mathrm{C}}$ can interact with $\mathrm{Hsp} 60$ and may have an important role in the binding and internalization of B. abortus into $\mathrm{M}$ cells. ${ }^{47}$ Thus, $\mathrm{PrP}^{\mathrm{C}}$ may also act as an uptake receptor for pathogenic bacteria, which express Hsp60.

\section{Other M-cell-specific proteins}

A number of other M-cell-specific proteins have been reported that may similarly selectively bind to components of potentially pathogenic microorganisms. However, experiments are required to determine their in vivo functions in $\mathrm{M}$ cells. ANXA5 is expressed by immature and mature $\mathrm{M} \mathrm{cells}^{48}$ and can bind to Gram-negative bacteria with high affinity via the lipid A domain of lipopolysaccharide (LPS).$^{49}$ The endotoxin activity of LPS is blocked when bound to ANXA5, implying an important anti-inflammatory role to protect the host against LPS-mediated endotoxemia. Alternatively, it is plausible that ANXA5 on M cells by binding LPS acts as an uptake receptor for Gram-negative bacteria. However, further studies are necessary to determine whether the lipid A domain of LPS would be directly accessible to M-cell-associated membrane receptors or whether lipid A only becomes accessible after bacterial degradation. Peptidoglycan recognition protein (PGLRP)-1 (encoded by Pglyrp1, also known as PGRP-S) is an innate pattern recognition protein that binds to bacterial peptidoglycan and is expressed highly by $\mathrm{M}$ cells. ${ }^{8}$ Little is known of the in vivo functions of four of the mammalian PGLRPs, but PGLRP1 has bactericidal activity against Grampositive bacteria and is not known to be involved in cell signaling. ${ }^{50}$ Whether the PGLRP1 secreted by M cells exhibits a similar bactericidal activity to help protect the host against internalized bacteria remains to be determined. The potential roles of other M-cell expressed proteins are less discernible, such as fatty acid-binding protein $5,{ }^{51}$ clusterin, ${ }^{52}$ and secretogranin V (encoded by Scg5, also known as Sgne-1). ${ }^{24}$

A phage-display screen of potential M-cell ligands identified one peptide, termed "Co1", which shared high sequence 
homology with the $\alpha 1$ helix of the outer membrane protein $\mathrm{H}$ $(\mathrm{OmpH})$ of Yersinia enterocolitica. ${ }^{53}$ The same region of $\mathrm{OmpH}$ is also homologous to that of an E. coli chaperone protein, Skp, which can act as a complement C5a receptor (C5aR) chemotactic ligand. Data from the combined analysis of an in vitro $\mathrm{M}$-cell-like model and mouse Peyer's patch $\mathrm{M}$ cells in vivo have suggested that $\mathrm{OmpH} \beta 1 \alpha 1$ binds to $\mathrm{C} 5 \mathrm{aR}$ on the apical M-cell surface and enhances the transcytosis of antigens into $\mathrm{M}$ cells. ${ }^{54}$ However, whether direct molecular interactions between $\mathrm{OmpH} \beta 1 \alpha 1$ and $\mathrm{C} 5 \mathrm{aR}$ (and homologous ligands) occur on $\mathrm{M}$ cells is uncertain.

\section{Secretory immunoglobulin A (IgA)}

Secretory IgA provides a first line of defence in the mucosal immune system by preventing the access of specific pathogens and their toxins across the intestinal epithelium, and is an important regulator of the composition of the commensal gut microflora. ${ }^{55}$ For example, secretory IgA is essential for protection from the effects of lumenal cholera toxin exposure. ${ }^{56}$ Secretory IgA can block lumenal pathogens from binding to gut epithelial cells and in doing so can provide protection against intestinal reovirus infection. ${ }^{57}$ Similarly, secretory IgA may also mediate the lumenal trapping of certain bacteria such as Shigella flexneri retaining them within the mucus layer overlying the intestinal epithelium. ${ }^{58}$ Prevention of infection against oral infection with $S$. Typhimurium has also been described. ${ }^{59}$ The targeting of secretory IgA-containing immune complexes to $\mathrm{M}$ cells appears to lead to their subsequent transcytosis through to the MNP and lymphocytes in the SED. ${ }^{60,61}$ Thus, in addition to the role of secretory IgA in facilitating the immune exclusion of lumenal pathogens and their antigens, the transcytosis of IgA by $M$ cells may also aid antigen sampling and modulate mucosal immune responses. Whether the binding and uptake of secretory IgA and/or IgA-containing immune complexes by $M$ cells occurs specifically is uncertain. No putative IgA receptor has been reported to be expressed by $\mathrm{M}$ cells. The possibility therefore cannot be excluded that the enhanced pinocytic and transcytotic activity of $\mathrm{M}$ cells alone is sufficient to efficiently internalize secretory $\operatorname{IgA}$ and/or IgA-containing immune complexes. Following their uptake from the gut lumen, IgAcontaining immune complexes do not appear to disseminate beyond the mesenteric lymph nodes. ${ }^{61}$ It is plausible that the secretory IgA-mediated, selective delivery of potential gut pathogens to Peyer's patches may act to enable antigen recognition under neutralizing and non-inflammatory conditions without causing widespread tissue dissemination.

\section{ATTRACTION TO, AND COMMUNICATION WITH, IMMUNE CELLS IN THE BASOLATERAL POCKET}

The M-cell basolateral pocket is a specialized microenvironment beneath the FAE that enables close interactions between M cells, MNP, B cells, and T cells. ${ }^{7,8}$ These cellular associations ensure that transcytosed microorganisms or macromolecules are readily acquired by specific immune cell populations to induce an appropriate immune response. $\mathrm{M}$ cells may even establish intercellular communication channels in order to transport cellular components between themselves and the cells within the basolateral pocket. ${ }^{62}$ Tunneling nanotubes (TNTs) are thin membranous intercellular conduits that enable the transport of cellular components between cells. Pathogens may also exploit these TNT conduits to aid their dissemination between host cells $s^{63,64}$ or to manipulate the host's response to infection by shuttling pathogen-derived immunosuppressive factors from infected cells to immune cell populations. ${ }^{65}$ TNFAIP2 (also known as M-Sec) is expressed specifically by mature $\mathrm{M}$ cells in a Spi-B-dependent manner. ${ }^{2,24}$ In the Peyer's patches, TNFAIP2 is associated with TNT-like structures, which appear to interconnect the $\mathrm{M}$ cells in the FAE. ${ }^{62}$ Data show that TNFAIP2 acts as a key regulator of TNT formation between immune cells. Thus, the close association of $\mathrm{M}$ cells with MNP raises the possibility that some microorganisms or macromolecules may be directly transferred from the gut lumen to MNP via M-cell-derived TNTs.

Chemokines and chemokine receptors have important roles in attracting lymphocytes and leukocytes to lymphoid tissues and controlling their positioning within them. In addition to CCL20, ${ }^{34}$ several other chemokines are also expressed by the FAE, some of them specifically by M cells, including CCL $9^{2,25,66}$ and $\mathrm{C}-\mathrm{X}-\mathrm{C}$ motif chemokine ligand 16 (CXCL16) (25,67 $^{25}$ (Figure 3). Retrospective analysis of the magnitude of all the chemokine-encoding genes expressed by microarray data sets derived from Peyer's patch $\mathrm{M}$ cells, ${ }^{25}$ the $\mathrm{FAE}^{26}$ and RANKL-stimulated villous epithelia ${ }^{2}$ also indicates significant expression of CXCL11 when compared with the small intestine villous epithelium. The detection of a specific set of chemokines implies that their expression is regulated by an underlying transcriptional program.

The wide range of chemokines and receptors expressed in mammals can lead to redundancy whereby several chemokines may bind and activate certain chemokine receptors. However, despite this apparent redundancy, mice deficient in the sole receptor for CCL20 (CCR6 $^{-1-}$ mice) show a significant deficiency in M-cell maturation in the FAE. ${ }^{36,37}$ These data suggest that the other FAE- and M-cell-expressed chemokines most likely have accessory roles, such as the attraction of specific cell populations to the basolateral pocket to sample antigens and mount an immune response. The chemokine CCL9 appears to be preferentially expressed by mature M cells and may aid the recruitment of $\mathrm{CD}_{11 \mathrm{~b}^{+}}$MNP to the SED of Peyer's patches. ${ }^{2,66}$ CXCL16 expression may promote interactions between the FAE and CXCR6-expressing T cells ${ }^{67}$ and may plausibly also act as an antimicrobial peptide in the mucosal epithelium. ${ }^{68}$ CCL25 expression may help mediate the homing of IgA antibody-secreting cells, ${ }^{69}$ whereas CXCL11 may stimulate the attraction of CXCR3-expressing Th1 cells. ${ }^{70}$

\section{PATHOGENS CAN EXPLOIT M CELLS TO INVADE THE HOST}

Pathogenic microorganisms have evolved a wide range of mechanisms to evade or even exploit immune cells to infect the host. $\mathrm{M}$ cells are no exception to this as their efficient transcytotic activity is exploited by numerous pathogens to breach the gut epithelium. In the absence of $\mathrm{M}$ cells, oral 
infections with $Y$. enterocolitica, ${ }^{36}$ prions, ${ }^{71}$ or retrovirus ${ }^{33}$ are blocked or impeded. A role for M cells as portals for the entry of L. monocytogenes, ${ }^{72}$ Mycobacterium avium subsp. paratuberculosis, ${ }^{73,74}$ S. Typhimurium, ${ }^{75}$ HIV type $1,{ }^{76}$ influenza virus, ${ }^{77}$ polio virus, ${ }^{78}$ and reovirus ${ }^{79}$ have also been described. Staphylococcus aureus secretes a number of virulence factors such as the enterotoxins, which can help subvert the host immune system. Data from in vitro studies imply that $\mathrm{M}$ cells may also aid the passage of staphylococcal enterotoxin A across the gut epithelium. ${ }^{80}$ Together, these examples show that whereas the sampling of intestinal bacteria by $M$ cells may be important for the induction of specific immunity in the Peyer's patches, this function has the potential to be a critical Achilles heel by enabling the safe passage of some pathogenic microorganism or their toxins across mucosal epithelia.

\section{PATHOGENS CAN MANIPULATE M CELLS TO INVADE THE HOST}

Although some pathogenic microorganisms may use molecules expressed on the apical surfaces of $\mathrm{M}$ cells as invasion receptors to infect the host, others appear to have evolved mechanisms that exploit the plasticity of enterocytes to specifically induce M-cell differentiation in the gut epithelium. For example, apparent increases in M-cell-density or antigen transcytosis in the FAE have been described following exposure to S. Typhimurium ${ }^{81}$ and Streptococcus pneumoniae R36a. ${ }^{82,83}$

The RANKL- and Spi-B-dependent differentiation of M cells from immature precursors in the crypts typically takes approximately 4 days, ${ }^{2,84}$ yet the studies above suggest that some pathogenic bacteria can achieve this within hours of exposure. ${ }^{81-83}$ These characteristics suggest that it is highly unlikely that terminally differentiated enterocytes or crypt stem cells could be converted by bacteria into mature $\mathrm{M}$ cells in such a short time frame. A more plausible explanation is that the bacteria produce factors which act on cells in the FAE that are already destined to become functionally mature M cells (e.g., immature, differentiating $M$ cells). Recent data support this hypothesis and show how a specific factor secreted by $S$. Typhimurium is used by the bacterium to enable the rapid trans-differentiation of FAE enterocytes into $\mathrm{M}$ cells to permit host invasion. ${ }^{3}$ Specifically, the SPI-1 encoded type III effector protein SopB secreted by $S$. Typhimurium induced the epithelial-mesenchymal transition of FAE enterocytes into $\mathrm{M}$ cells. This cellular trans-differentiation occurred via the SopB-dependent activation of $\mathrm{Wnt} / \beta$-catenin signalling and led to the induction of both RANKL and its receptor RANK. The autocrine RANKL-RANK activation of the cells induced the expression of Spi-B and also the epithelial-mesenchymal transition-regulating transcription factor Slug (encoded by Snai2) that triggered the FAE enterocytes to trans-differentiate into $\mathrm{M}$ cells. ${ }^{3}$

\section{AGING IMPEDES THE FUNCTIONAL MATURATION OF M CELLS}

The mucosal immune response in the intestine is significantly compromised by aging. ${ }^{85-88}$ This age-related decline in immune competence is associated with diminished antigenspecific IgA antibody titers in the intestinal lumen ${ }^{86,89}$ and a decreased ability to generate tolerance to harmless antigens. ${ }^{87}$ Despite the important role of $\mathrm{M}$ cells in mucosal immunity in the Peyer's patches, nothing was known about how aging influenced their development and function. Using an immunosenescent mouse model, we have recently shown that there is a dramatic decline in the functional maturation of $\mathrm{GP} 2{ }^{+} \mathrm{M}$ cells in the Peyer's patches of aged mice ( $\geqslant 600$ days old). Furthermore, the transcytosis of particulate antigen across the FAE was likewise impeded. ${ }^{38}$ In order to understand the mechanism underlying this aging-related decline in M-cell status, we also examined the effects on the stimuli responsible for their induction and maturation. Aging did not affect RANKL expression by the SED stromal cells or the initial induction of $\mathrm{M}$ cell differentiation but specifically impaired the expression of Spi-B and downstream functional maturation of $\mathrm{GP}^{+} \mathrm{M}$ cells. Expression of the chemokine CCL20 was also reduced in the FAE of aged Peyer's patches, consistent with data from an independent study of the effects of aging on isolated lymphoid follicles. ${ }^{90}$ As a consequence of the impaired CCL20 expression, the attraction cells (including the "M-cell-inducing" CD11c ${ }^{+} \mathrm{B}$ cells ${ }^{37}$ ) towards the FAE was impaired. As both the M-cell intrinsic expression of Spi-B $\mathrm{B}^{2,15,22}$ and CCL20CCR6 stimulation ${ }^{36,37}$ each have important roles in M-cell differentiation, the effects of aging on Spi-B and CCL20 expression appear to dramatically impede the functional maturation of $\mathrm{M}$ cells.

Further studies are required to determine whether this agingrelated deficiency in the mucosal immune system's ability to sample lumenal antigens contributes to the aging-related decline in impaired antigen-specific mucosal immune responses. ${ }^{86}$ However, as several pathogens such as S. Typhimurium ${ }^{75}$ and prions ${ }^{71}$ (as described above) exploit $M$ cells to invade the host, the effects of ageing on M-cell status may also paradoxically have unexpected protective benefits. For example, the effects of aging on M-cell status may impede the early M-cell-dependent uptake of these pathogens into the Peyer's patches and in doing so significantly reduce disease susceptibility. ${ }^{91,92}$

\section{M-CELL-BINDING LIGANDS AS NOVEL MUCOSAL VACCINE TARGETS}

The significant advances that have been made in the identification of M-cell-specific surface markers or "immunosurveillance" receptors have focussed attention on the development systems to specifically deliver antigens to $M$ cells in order to improve the efficacy of mucosal vaccines. A number of examples are discussed below.

\section{Lectins}

The lectin UEA-1 binds to sites of $\alpha-1,2$ fucosylation on the surfaces of $M$ cells with high affinity. Initial attempts using UEA-1 to target antigen to M cells showed that such approaches were effective in inducing antigen-specific mucosal IgA responses. ${ }^{93-96}$ Along a similar theme, oral vaccination with 
antigen-encapsulated liposomes coupled to UEA-1 (termed lectinized liposomes) also led to increased uptake by Peyer's patch $\mathrm{M}$ cells and the induction of higher antigen-specific secretory IgA responses. ${ }^{97}$

Alternative approaches to target antigens to the $\alpha(1,2)$ fucose-containing carbohydrate moiety on $\mathrm{M}$ cells have also been explored, for example, by using the non-toxic, smallmolecular UEA-1 mimetic, tetragalloyl-D-lysine dendrimer. ${ }^{98}$ The mAb NKM 16-2-4 specifically recognizes the $\alpha(1,2)$ fucose-containing carbohydrate moiety on $\mathrm{M}$ cells with a greater affinity than that of UEA-1. Oral vaccine formulations comprising antigen coupled to mAb NKM 16-2-4 (with cholera toxin as a mucosal adjuvant) have been shown to be effective in inducing high antigen-specific mucosal IgA responses. Furthermore, oral vaccination with botulinum toxinconjugated mAb NKM 16-2-4 induced protective immunity against oral exposure to a lethal toxin dose. ${ }^{29}$

\section{Claudin 4}

Although claudin 4 is a component of tight junctions, this protein may also be redistributed to the cytoplasm of $\mathrm{M}$ cells and have a role in endocytosis. A peptide derived from the C-terminal domain of the Clostridium perfringens enterotoxin binds to the external domain of claudin 4 and can mediate uptake by $\mathrm{M}$ cells in vivo. ${ }^{99,100}$ Furthermore, this claudin 4binding peptide when coupled to antigen is effective in enhancing mucosal antigen-specific secretory IgA responses after intranasal immunization. ${ }^{100}$

\section{C5aR ligands}

As described above, the M-cell ligand "Co1" shared high sequence homology with $\mathrm{OmpH}$ of $Y$. enterocolitica, and each of these peptides appeared to bind to a C5aR on the apical surface of M cells. ${ }^{54}$ The conjugation of antigens to either Col or OmpH could mediate their delivery to M cells in the Peyer's patches and induce enhanced mucosal immune responses after oral administration. ${ }^{53,54}$

\section{Reovirus hemagglutinin protein $\sigma 1$}

DNA vaccines have also been shown to confer protective immunity against a range of pathogenic microrganisms, but this approach has proved to be poor at inducing mucosal immunity. M-cell targeting has also been explored to attempt to improve the efficiency of the delivery of a DNA vaccine to a mucosal-inductive tissue. As reovirus is considered to bind $\mathrm{M}$ cells in vivo, ${ }^{79}$ a recombinant reovirus hemagglutinin protein $\sigma 1$ conjugated to polylysine was used to target a DNA vaccine to NALT M cells. ${ }^{101}$ Intranasal immunization with protein $\sigma 1$-polylysine DNA was effective in inducing prolonged antigen-specific mucosal IgA responses, illustrating that M-cell-targeted peptide or DNA vaccinations may each represent effective means of inducing antigen-specific mucosal immunity.

However, in addition to their role in the induction of specific mucosal immunity, $M$ cells may also be involved in the induction of oral tolerance. Independent studies show that mucosal administration of low doses of reovirus protein $\sigma 1$ conjugated to ovalbumin induced systemic unresponsiveness and failed to elicit an ovalbumin-specific mucosal immune response. Under these conditions, the mice were shown to be tolerogenic to ovalbumin and were resistant to peripheral challenge due to the generation of antigen-specific regulatory $\mathrm{T}$ cells. ${ }^{102,103}$

\section{CONCLUDING REMARKS}

Significant recent advances have been made in the identification of M-cell-specific surface markers and the characterization of the range of pathogen-derived ligands these "immunosurveillance" receptors can bind. By using similar approaches to those described above, it may be possible to significantly improve the efficacy and specificity of the M-cell targeting and elicit long-lived and protective mucosal immunity or tolerance. The identification of the molecular mechanisms that some pathogens use to exploit $\mathrm{M}$ cells to invade the host may also lead to the development of specific strategies to block the initial host colonization. Although the targeted delivery of vaccine antigens to $\mathrm{M}$ cells offers potential as an effective means of inducing antigen-specific mucosal immune responses, the observation that the functional maturation of $\mathrm{M}$ cells is dramatically impaired in aged mice implies that these strategies may be much less effective when used to immunize the elderly. ${ }^{38} \mathrm{An}$ understanding of the molecular mechanism that underpins this aging-related decline in M-cell status will help identify novel approaches to stimulate M-cell differentiation and potentially improve mucosal immunity in the elderly.

The use of various M-cell-deficient mouse models has greatly improved our understanding of the immunobiology of $\mathrm{M}$ cells in immunity and disease pathogenesis in vivo. However, in many of these instances, the possibility that M-cell-independent factors significantly contribute to the effects described cannot be entirely excluded. For example, RANKL neutralization dramatically depletes $\mathrm{M}$ cells in the FAE in vivo. ${ }^{20}$ However, RANKL may influence gene expression by other cells in the FAE, ${ }^{13}$ which may also have a role in the uptake of lumenal antigens. ${ }^{104}$ Similarly, the maturation of $\mathrm{M}$ cells is impaired in the FAE of CCR6 $6^{-1-}$ mice, ${ }^{36-38}$ but the effects on mucosal immunity or disease susceptibility due to the reduced size of their Peyer's patches or defective T-cell responses cannot be entirely excluded. The independent demonstrations that expression of the transcription factor Spi-B is critical for the functional maturation of $\mathrm{M}$ cells $\mathrm{s}^{2,15,22}$ has identified the Spi- $\mathrm{B}^{-1-}$ mouse as an alternative approach to study the immunobiology of $\mathrm{M}$ cells. However, Spi-B also has an important role in the differentiation of plasmacytoid DC and B cells. ${ }^{105,106}$ In order to completely avoid these off-target effects, conditional knock-out mice with genes encoding RANK or Spi-B specifically ablated in the intestinal epithelium are crucial to study mucosal immunity in the specific absence of $M$ cells.

An in vitro M-cell-like model system in which a monolayer of the differentiated human enterocyte cell line Caco-2 is cultivated with B cells has been widely used to study $\mathrm{M}$ cells. ${ }^{32}$ However, although these cells display efficient transcytotic 
Table 1 Comparison of M-cell-related genes expressed in the villous epithelium in response to RANKL-stimulation, RANKLstimulated in vitro intestinal organoids and in vitro M-cell-like differentiated Caco-2 cells

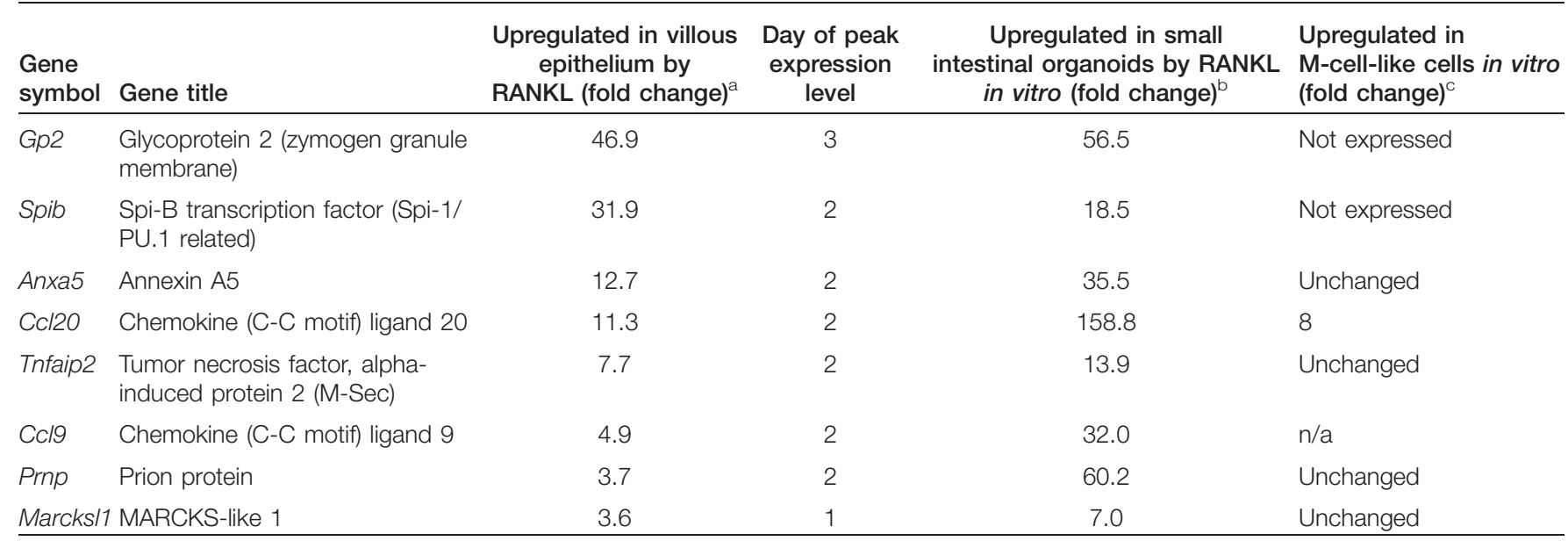

$\mathrm{n} / \mathrm{a}$, an equivalent annotated probe set was not present on the array; MARCKS, myristoylated alanine-rich C-kinase substrate; RANKL, receptor activator of nuclear factor- $\kappa \mathrm{B}$ ligand.

These data (GSE37861) $)^{2}$ were performed on Affymetrix mouse gene 1.0 STexpression arrays. Fold change in gene expression level at peak day when compared with controls (day 0).

${ }^{b}$ These data were performed on Agilent $4 \times 44 \mathrm{~K}$ whole-mouse genome expression arrays (Agilent Technologies, Inc., Santa Clara, CA) and equivalent gene probe sets were compared (GSE38785). ${ }^{15}$ Fold change in gene expression when compared with unstimulated control organoids.

${ }^{c}$ Performed on Affymetrix human genome U133 plus 2 expression arrays and equivalent gene probe sets were compared. Fold change in gene expression by M-cell-like differentiated Caco-2 cells when compared with undifferentiated controls (GSE25330, GSE7745). ${ }^{115}$

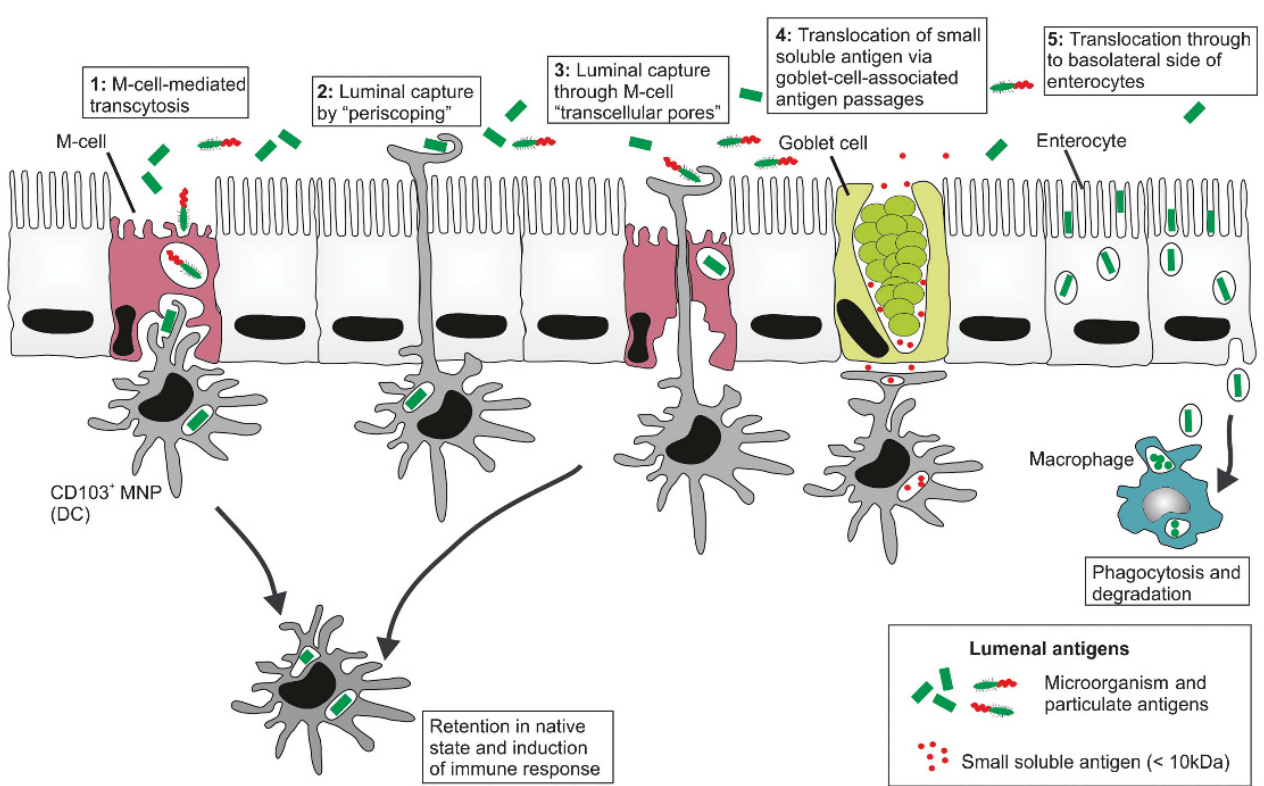

Figure 4 Potential routes of lumenal antigen sampling in the intestine by epithelial cell subsets and mononuclear phagocytes (MNP). (1) Sampling of gut lumenal microorganisms and macromolecules via M-cell-mediated transcytosis. (2) Although the incidence is rare in the steady state, certain inflammatory stimuli appear to recruit MNP from the lamina propria where they insert their dendrites through the tight junctions between enterocytes to directly sample the luminal contents. ${ }^{108,109}$ (3) Another study has reported that MNP may even sample lumenal antigens by extending their dendrites directly through "transcellular pores" in the M cells themselves. ${ }^{7}(4)$ Mucin-secreting goblet cells can also function as passages for the delivery of lowmolecular weight soluble antigens to $\mathrm{CD}_{103^{+}}$MNP in the lamina propria. ${ }^{107}$ (5) Ultrastructural analysis suggests that follicle-associated epithelia enterocytes can also acquire certain gut lumenal antigens and exocytose the intravascular contents of their large late endosomes into the extracellular space of the subepithelial dome where they are acquired by MNP. ${ }^{104} \mathrm{DC}$, dendritic cell.

activity in contrast to the undifferentiated enterocytes, it is uncertain whether these co-cultivated epithelial cells accurately represent the many characteristics of $\mathrm{M}$ cells in vivo. Furthermore, analysis of the genes expressed by "M-cell induced" Caco- 2 cells shows that while they express
CCL20 highly, they lack expression of many mature M-cell marker genes, such as GP2 and SPIB (Table 1). Recently, exciting progress was reported on the development of a novel, potentially physiologically relevant in vitro M-cell-model system. In this system, RANKL stimulation induces M-cell 
differentiation in gut organoid cultures established from intestinal crypts or single Lgr $^{+}$crypt stem cells. ${ }^{15}$ As well as exhibiting high transcytotic activity, the range of genes expressed by these organoid cultures closely resembles those of $\mathrm{M}$ cells in vivo (Table 1). These M-cell-containing organoid cultures offer a simple and physiologically relevant in vitro model system in which to study the immunobiology of $\mathrm{M}$ cells from a range of mammalian species.

Finally, many studies have primarily focussed on $\mathrm{M}$ cells as the main sites of antigen sampling in mucosal epithelia, but several studies are challenging the assertion that this activity is so restricted (Figure 4). Data from the ultrastructural analysis of Peyer's patches identified a novel, previously unrecognized, potentially important component of the normal machinery for antigen sampling in the GALT. This study suggested that FAE enterocytes can also acquire certain gut lumenal antigens and exocytose the intravascular contents of their large late endosomes into the extracellular space of the SED where they are acquired by MNP. ${ }^{104}$ Goblet cells are situated throughout mucosal epithelia and, until recently, were mainly considered to function as mucin-secreting cells. However, an elegant study shows that intestinal goblet cells can also function as passages for the delivery of low-molecular weight soluble antigens to $\mathrm{CD}_{103}{ }^{+} \mathrm{MNP}$ in the lamina propria. ${ }^{107}$ The sampling of lumenal antigens may also not be entirely restricted to epithelial cell populations. Although the incidence is rare in the steady state, certain inflammatory stimuli appear to recruit MNP from the lamina propria to the gut epithelium where they insert their dendrites through the tight junctions between enterocytes to directly sample the luminal contents. ${ }^{108,109}$ In addition to this "periscoping" activity, another study has even proposed that MNP sample lumenal antigens by extending their dendrites directly through "transcellular pores" in the M cells themselves. ${ }^{7}$

The precise immunological consequences of these alternative antigen sampling mechanisms are uncertain and require further analysis. Unlike macrophages that rapidly phagocytose microorganisms and destroy them in their phagosomes, classical DC appear to be equipped with both degradative and nondegradative antigen uptake pathways to facilitate antigen presentation to both $\mathrm{T}$ and $\mathrm{B}$ cells. ${ }^{110,111}$ Indeed, classical DC can capture and retain unprocessed antigen in vitro and in vivo and can transfer this antigen to naive B cells to initiate a specific antibody response. ${ }^{112}$ Viable commensal bacteria and unprocessed antigen can also be recovered from classical DC migrating within afferent mesenteric lymph. ${ }^{113,114}$ These contrasting characteristics raise the question of whether the cells through which antigens are initially sampled across the intestinal epithelium ( $M$ cells, enterocytes, goblet cells) determine whether lumen antigens are initially transferred directly into the Peyer's patches (such as after M-cell-mediated transcytosis) or into villi and onwards to the mesenteric lymph nodes. Similarly, it is also plausible that in the steady-state these routes also direct lumenal antigens to specific MNP populations and, as a consequence, fundamentally influence the nature of the immune response directed to those antigens.

\section{ACKNOWLEDGEMENTS}

This work was supported by project (BB/J014672/1; BB/K021257/1) and Institute Strategic Programme Grant (BB/J0004332/1) funding from the Biotechnology and Biological Sciences Research Council. I.R.W. was supported by grant funding from the National Institutes of Health (DK064730). We thank Dr Atsushi Kobayashi (Tohoku University Graduate School of Medicine, Sendai, Japan) for immunohistochemistry expertise.

\section{DISCLOSURE}

The authors declared no conflict of interest.

(c) 2013 Society for Mucosal Immunology

\section{REFERENCES}

1. Lorenz, R.G. \& Newberry, R.D. Isolated lymphoid follicles can function as sites for induction of mucosal immune responses. Ann. New York Acad. Sci. 1029, 44-57 (2004).

2. Kanaya, T. et al. The Ets transcription factor Spi-B is essential for the differentiation of intestinal microfold cells. Nat. Immunol. 13, 729-736 (2012).

3. Tahoun, A. et al. Salmonella transforms follicle-associated epithelial cells into M cells to promote intestinal invasion. Cell Host Microbe 12, 645-666 (2012).

4. Mabbott, N.A., Baillie, J.C., Hume, D.A. \& Freeman, T.C. Meta-analysis of co-expressed gene signatures in mouse leukocyte populations. Immunobiology 215, 724-736 (2010).

5. Bradford, B.M., Sester, D., Hume, D.A. \& Mabbott, N.A. Defining the anatomical localisation of subsets of the murine mononuclear phagocyte system using integrin alpha $\mathrm{X}$ (ITGAX) and colony stimulating factor 1 receptor (CSF1-R) expression fails to discriminate dendritic cells from macrophages. Immunobiology 216, 1228-1237 (2011).

6. Hume, D.A., Mabbott, N., Raza, S. \& Freeman, T.C. Can DCs be distinguished from macrophages by molecular signatures. Nat. Immunol. 14, 187-189 (2013).

7. Lelouard, H., Fallet, M., De Bovis, B., Meresse, S. \& Gorvel, J.P. Peyer's patch dendritic cells sample antigens by extending dendrites through $M$ cell-specific transcellular pores. Gastroenterology 142, 592-601 (2012).

8. Wang, J., Gusti, V., Saraswati, A. \& Lo, D.D. Convergent and divergent development among $\mathrm{M}$ cell lineages in mouse mucosal epithelium. J. Immunol. 187, 5277-5285 (2011).

9. Hase, K. et al. Uptake through glycoprotein $2 \mathrm{ofFimH}^{+}$bacteria by M cells initiates mucosal immune responses. Nature 462, 226-231 (2009).

10. Kim, D.Y. et al. The airway antigen sampling system: respiratory $M$ cells as an alternative gateway for inhaled antigens. J. Immunol. 186, 4253-5262 (2011).

11. Vallon-Eberhard, A., Landsman, L., Yogev, N., Verrier, B. \& Jung, S. Transepithelial pathogen uptake into the small intestinal lamina propria. J. Immunol. 176, 2465-2469 (2006).

12. Jang, M.H. et al. Intestinal villous $M$ cells: An antigen entry site in the mucosal epithelium. Proc. Natl. Acad. Sci. USA 101, 6110-6115 (2004).

13. Kobayashi, A. et al. Identification of novel genes selectively expressed in the follicle-associated epithelium from the meta-analysis of transcriptomics data from multiple mouse cell and tissue populations. DNA Res. 19, 407-422 (2012).

14. Sato, T. et al. Single Lgr5 + stem cells build crypt-villus structures in vitro without a mesenchymal niche. Nature 459, 262-265 (2009).

15. de Lau, W. et al. Peyer's patch M cells derive from Lgr5 + stem cells, require SpiB and are induced by RankL in cultured 'organoids'. Mol. Cell Biol. 32, 3639-3647 (2012).

16. Lopez-Garcia, C., Klein, A.M., Simmons, B.D. \& Winton, D.J. Intestinal stem cell replacement follows a pattern of neutral drift. Science $330,822-$ 825 (2010).

17. Snippert, H.J. et al. Intestinal crypt homeostasis results from neutral competition between symetrically dividing Lgr5 stem cells. Cell 143, 134-144 (2010).

18. Taylor, R.T. et al. Lymphotoxin-independent expression of TNF-related activation-induced cytokine by stromal cells in cryptopatches, isolated lymphoid follicles, and Peyer's patches. J. Immunol. 178, 5659-5667 (2007). 
19. Katakai, T. et al. Organizer-like reticular stromal cell layer common to adult secondary lymphoid organs. J. Immunol. 181, 6189-6200 (2008).

20. Knoop, K.A. et al. RANKL is necessary and sufficient to initiate development of antigen-sampling $\mathrm{M}$ cells in the intestinal epithelium. J. Immunol. 183, 5738-5747 (2009).

21. Trouvin, A.-P. \& Goeb, V. Receptor activator of nuclear factor-кB ligand and osteoprotegerin: maintaining the balance to prevent bone loss. Clin. Intervent. Aging 5, 345-354 (2010).

22. Sato, S. et al. Transcription factor Spi-B-dependent and -independent pathways for the development of Peyer's patch M cells. Mucosal Immunol. 6, 838-846 (2013).

23. Lo, D. et al. Cell culture modelling of specialized tissue: identification of genes expressed specifically by follicle-associated epithelium of Peyer's patch by expression of profiling of Caco-2/Raji co-cultures. Int. Immunol. 16, 91-99 (2004)

24. Hase, K. et al. Distinct gene expression profiles characterize cellular phenotypes of follicle-associated epithelium and M cells. DNA Res. 12, 127-137 (2005).

25. Terahara, K. et al. Comprehensive gene expression profiling of Peyer's patch M cells, villous M-like cells, and intestinal epithelial cells. J. Immunol. 180, 7840-7846 (2008).

26. Nakato, G. et al. New approach for M-cell-specific molecules by screening comprehensive transcriptome analysis. DNA Res. 16, 227-235 (2009).

27. Kenis, $\mathrm{H}$. et al. Cell surface-expressed phosphatidylserine and annexin A5 open a novel portal of cell entry. J. Biol. Chem. 279, 52623-52629 (2004).

28. Bjorkblom, B. et al. C-Jun N-terminal kinase phosphorylation of MARCKSL1 determines actin stability and migration in neurons and in cancer cells. Mol. Cell. Biol. 32, 3513-3526 (2012).

29. Nochi, T. et al. A novel M cell-specific carbohydrate-targeted mucosal vaccine effectively induces antigen-specific immune responses. J. Exp. Med. 204, 2789-2796 (2007).

30. Hsieh, E.H. \& Lo, D.D. Jagged1 and notch1 help edit M cell patterning in Peyer's patch follicle epithelium. Dev. Comp. Immunol. 27, 306-312 (2012).

31. Onishi, S. et al. Immunohistochemical study on the delayed progression of epithelial apoptosis in follicle-associated epithelium of rat Peyer's patch. J. Vet. Med. Sci. 69, 112301129 (2007).

32. Kerneis, S., Bogdanova, A., Kraehenbuhl, J.-P. \& Pringualt, E. Conversion by Peyer's patch lymphocytes of human enterocytes into $M$ cells that transport bacteria. Science 277, 949-952 (1997).

33. Golovkina, T.V., Schlomchik, M., Hannum, L. \& Chervonsky, A. Organogenic role of B lymphocytes in mucosal immunity. Science 286, 1965 (1999)

34. Iwasaki, A. \& Kelsalla, B.A. Localization of distinct Peyer's patch dendritic cell subsets and their recruitment by chemokines macrophage inflammatory protein (MIP)-3, MIP-3ß, and secondary lymphoid organ chemokine. J. Exp. Med. 191, 1381-1394 (2000).

35. Cook, D.N. et al. CCR6 mediates dendritic cell localization, lymphocyte homeostasis, and immune responses in mucosal tissue. Immunity 12 495-503 (2000).

36. Westphal, S. et al. Resistance of chemokine receptor 6-deficient mice to Yersinia enterocolitica infection: evidence on defective M-cell formation in vivo. Am. J. Pathol. 172, 671-680 (2008).

37. Ebisawa, M. etal. CCR $6^{\text {hi }} C D 11 c^{\text {int }} B$ cells promote M-cell differentiation in Peyer's patch. Int. Immunol. 23, 261-269 (2011).

38. Kobayashi, A. et al. The functional maturation of $\mathrm{M}$ cells is dramatically reduced in the Peyer's patches of aged mice. Mucosal Immunol., in press (2013).

39. Sierro, F. et al. Flagellin stimulation of intestinal epithelial cells triggers CCL20-mediated migration of dendritic cells. Proc. Natl. Acad. Sci. USA 98, 13722-13727 (2001).

40. Hsieh, E.N. et al. CD137 is required for M cell functional maturation but not lineage commitment. Am. J. Pathol. 177, 666-676 (2010).

41. Man, A.L. et al. Macrophage migration inhibitory factor plays a role in the regulation of microfold (M) cell-mediated transport in the gut. J. Immunol. 181, 5673-5680 (2008).

42. Rumbo, M., Sierro, F., Debard, N., Kraehenbuhl, J.P. \& Finke, D. Lymphotoxin beta receptor signaling induces the chemokine CCL2O in intestinal epithelium. Gastroenterology 127, 213-223 (2004).
43. Wang, J., Lopez-Fraga, M., Rynko, A. \& Lo, D.D. TNFR and LT $\beta R$ agonists induce follicle-associated epithelium and $\mathrm{M}$ cell specific genes in rat and human intestinal epithelial cells. Cytokine 47, 69-76 (2009).

44. Sirard, J.-C., Didierlaurent, A., Cayet, D., Sierro, F. \& Rumbo, M. Toll-like receptor 5- and lymphotoxin b receptor-dependent epithelial cell Ccl20

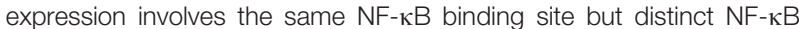
pathways and dynamics. Biochem. Biophys. Acta 1789, 386-294 (2009).

45. Tumanov, A.V., Kuprash, D.V., Mach, J.A., Nedospasov, S.A. \& Chervonsky, A.V. Lymphotoxin and TNF produced by B cells are despensable for maintenance of the follicle-associated epithelium but are required for development of lymphoid follicles in the Peyer's patches. J. Immunol. 173, 86-91 (2004).

46. Pak, J., Pu, Y., Zhang, Z.T., Hasty, D.L. \& Wu, X.R. Tamm-Horsfall protein binds to type 1 fimbriated Eschericia coli and prevents E. coli from binding to uroplakin la and lb receptors. J. Biol. Chem. 276, 9924-9930 (2001).

47. Nakato, G. et al. Cutting edge: Brucella abortus exploits a cellular prion protein on intestinal $\mathrm{M}$ cells as an invasive receptor. J. Immunol. 189, 1540-1544 (2012).

48. Verbrugghe, P. et al. Murine $M$ cells express annexin $V$ specifically. J. Pathol. 209, 240-249 (2006).

49. Rand, J.H. et al. Annexin A5 binds to lipopolysaccharide and reduces its endotoxin activity. mBio 3, e00292-00211 (2012).

50. Osanai, A. et al. Mouse peptidogylcan recognition protein PGLYRP-1 plays a role in the host innate immune response against Listeria monocytogenes infection. Infect. Immun. 79, 858-866 (2011).

51. Suzuki, R. et al. Localization of fatty acid binding protein of epidermal type common to dendritic cells and presumptive macrophages in Peyer's patches and epithelial $\mathrm{M}$ cells of mouse intestine. Histochem. Cell Biol. 132, 577-584 (2009).

52. Verbrugghe, P., Kujala, P., Waelput, W., Peters, P.J. \& Cuvelier, C.A. Clusterin in human gut-associated lymphoid tissue, tonsils, and adenoids: localization to M cells and follicular dendritic cells. Histochem. Cell Biol. 129, 311-320 (2008).

53. Kim, S.H., Seo, K.W., Kim, J., Lee, K.Y. \& Jang, Y.S. The M cell-targeting ligand promotes antigen delivery and induces antigen-specific immune responses in mucosal vaccination. J. Immunol. 185, 5787-5795 (2010).

54. Kim, S.H. et al. M cells expressing the complement C5a receptor are efficient targets for mucosal vaccine delivery. Eur. J. Immunol. 41, 32193229 (2011)

55. Mantis, N.J., Rol, N. \& Corthesy, B Secretory IgA's complex roles in immunity and mucosal homeostasis in the gut. Mucosal Immunol. 4, 603-611 (2011).

56. Lycke, N., Erlandsson, L., Ekman, L., Schon, K. \& Leanderson, T. Lack of $\mathrm{J}$ chain inhibits the transport of gut IgA and abrogates the development of intestinal antitoxic protection. J. Immunol. 163, 913-919 (1999).

57. Silvey, K.J., Hutchings, A.B., Vajdy, M., Petzke, M.M. \& Neutra, M.R. Role of immunoglobulin $A$ in protection against reovirus entry into murine Peyer's patches. J. Virol. 75, 10870-10879 (2001).

58. Boullier, S. et al. Secretory IgA-mediated neutralization of Shigella flexneri prevents intestinal tissue destruction by down-regulating inflammatory circuits. J. Immunol. 183, 5879-5885 (2009).

59. Michetti, P., Mahan, M.J., Slauch, J.M., Mekalanos, J.J. \& Neutra, M.R. Monoclonal secretory immunoglobulin A protects mice against oral challenge with the invasive pathogen Salmonella typhimurium. Infect. Immun. 60, 1786-1792 (1992).

60. Rey, J., Garin, N., Spertini, F. \& Corthesy, B. Targeting of secretory IgA to Peyer's patch dendritic cells and Tcells after transport by intestinal $\mathrm{M}$ cells. J. Immunol. 172, 3026-3033 (2004).

61. Kadaoui, K.A. \& Corthesy, B. Secretory IgA mediates bacterial translocation to dendritic cells in mouse Peyer's patches with restriction to mucosal compartment. J. Immunol. 179, 7751-7757 (2007).

62. Hase, K. etal. M-Sec promotes membrane nanotube formation by interacting with Ral and the exocyst complex. Nat. Cell. Biol. 11, 1427-1432 (2009).

63. Gousset, K. et al. Prions hijack tunnelling nanotubes for intercellular spread. Nat Cell. Biol. 11, 328-336 (2009).

64. Eugenin, E.A., Gaskill, P.J. \& Berman, J.W. Tunelling nanotubes (TNT) are induced by HIV-infection of macrophages: a potential mechanism for intercellular HIV trafficking. Cell. Immunol. 254, 142-148 (2009).

65. Xu, W. et al. HIV-1 evades virus-specific IgG2 and IgA responses by targeting systemic conduits and intestinal B cells via long-range intercellular conduits. Nat. Immunol. 10, 1008-1017 (2009). 
66. Zhao, X. et al. CCL9 is secreted by the follicle-associated epithelium and recruits dome region Peyer's patch $\mathrm{CD} 11 \mathrm{~b}^{+}$dendritic cells. J. Immunol. 171, 2797-2803 (2003).

67. Hase, K. et al. The membrane-bound chemokine CXCL16 expressed on follicle-associated epithelium and $M$ cells mediates lymph-epithelial interaction in GALT. J. Immunol. 176, 43-51 (2006).

68. Kotarsky, K. et al. A novel role for constitutively expressed epithelialderived chemokines as antibacterial peptides in the mucosa. Mucosal Immunol. 3, 40-48 (2010).

69. Hieshima, K. et al. CC chemokine ligands 25 and 28 play essential roles in intestinal extravasation of IgA antibody-secreting cells. J. Immunol. 173, 3668-3675 (2004)

70. Lee, J.W. et al. Differential regulation of chemokines by IL-17 in colonic epithelial cells. J. Immunol. 181, 6536-6545 (2008).

71. Donaldson, D.S. et al. M cell depletion blocks oral prion disease pathogenesis. Mucosal Immunol. 5, 216-225 (2012).

72. Chiba, S. et al. Listerial invasion protein internalin B promotes entry into ileal Peyer's patches in vivo. Microbiol. Immunol. 55, 123-129 (2011).

73. Ponnusamy, D., Periasamy, S., Tripathi, B.N. \& Pal, A. Mycobacterium avium subsp. paratuberculosis invades through $\mathrm{M}$ cell and enterocytes across ileal and jejunal mucosa of lambs. Res. Vet. Sci. 94, 306-312 (2012),

74. Bermundez, L.E., Petrofsky, M., Sommer, S. \& Barletta, R.G. Peyer's patch-deficient mice demonstrate that Mycobacterium avium subsp. paratuberculosis translocates across the mucosal barrier via both $\mathrm{M}$ cells and enterocytes but has inefficient dissemination. Infect. Immun. 78, 3570-3577 (2010).

75. Jones, B.D, Ghori, N. \& Falkow, S Salmonella typhimurium initiates murine infection by penetrating and destroying the specialized epithelial M cells of the Peyer's patches. J. Exp. Med. 180, 15-23 (1994).

76. Amerongen, H.M. et al. Transepithelial transport of HIV-1 by intestinal M cells: a mechanism for transmission of AIDS. J. Acquir. Immune Defic. Syndr. 4, 760-765 (1991).

77. Fujimura, Y. et al. The role of M cells of human nasopharyngeal lymphoid tissue in influenza virus sampling. Virchows Arch. 444, 36-42 (2004).

78. Takahashi, Y. et al. Nonhuman primate intestinal villous M-like cells: an effective poliovirus entry site. Biochem. Biophys. Res. Commun. 368, 501-507 (2008).

79. Wolf, J.L. et al. Intestinal M cells: a pathway for entry of reovirus into the host. Science 212, 471-472 (1981).

80. Maresca, M., Dumay, E., Fantini, J. \& Caporiccio, B. Selective transport of staphylococcal enterotoxin A through in vitro generated human $\mathrm{M}$ cells. Microbes Infect. 9, 1507-1510 (2007).

81. Savidge, T.C., Smith, M.W., James, P.S. \& Aldred, P. Salmonella-induced M-cell formation in germ-free mouse Peyer's patch tissue. Am. J. Pathol. 139, 177-184 (1991).

82. Borghesi, C. \& Nicoletti, C. Rapid appearance of M cells after microbial challenge is restricted at the periphery of the follicle-associated epithelium of Peyer's patch. Lab. Invest. 79, 1393-1401 (1999).

83. Meynell, H.M. et al. Up-regulation of microsphere transport across the follicle-associated epithelium of Peyer's patch by exposure to Streptococcus pneumoniae R36a. FASEB J. 13, 611-619 (1999).

84. Knoop, K.A., Butler, B.R., Kumar, N., Newberry, R.D. \& Williams, I.R. Distinct developmental requirements for isolated lymphoid follicle formation in the small and large intestine. Am. J. Pathol. 179, 18611871 (2011).

85. Schmucker, D., Owen, R., Outenreath, R. \& Thoreux, K. Basis for the agerelated decline in intestinal mucosal immunity. Clin. Dev. Immunol. 10, 167-172 (2003).

86. Koga, T. et al. Evidence for early aging in the mucosal immune system. J. Immunol. 165, 5352-5359 (2000).

87. Kato, H. et al. Lack of oral tolerance in ageing is due to sequential loss of Peyer's patch cell interactions. Int. Immunol. 15, 145-158 (2003).

88. Fujihashi, K. \& McGhee, J.R. Mucosal immunity and tolerance in the elderly. Mech. Ageing Dev. 125, 889-898 (2004).

89. Dunn-Walters, D.K., Banerjee, M. \& Mehr, R. Effects of age in antibody affinity maturation. Biochem. Soc. Trans. 31, 447-448 (2003).

90. McDonald, K.G., Leach, M.R., Huang, C., Wang, C. \& Newberry, R.D. Aging impacts isolated lymphoid follicle development and function. Immun. Aging 8, 1 (2011).
91. Ren, Z. et al. Effect of age on susceptibility to Salmonella typhimurium infection in C57BL/6 mice. J. Med. Microbiol. 58, 1559-1567 (2009).

92. Brown, K.L., Wathne, G.J., Sales, J., Bruce, M.E. \& Mabbott, N.A. The effects of host age on follicular dendritic cell status dramatically impair scrapie agent neuroinvasion in aged mice. J. Immunol. 183, 5199-5207 (2009).

93. Manocha, M. et al. Enhanced mucosal and systemic immune responses with intranasal immunization of mice with HIV peptides entrapped in PLG microparticles in combination with Ulex Europaeus-I lectin as $\mathrm{M}$ cell target. Vaccine 23, 5599-5617 (2005)

94. Wang, X. et al. Transgene vaccination using Ulex europaeus I (UEA-1) for targeted mucosal immunization against HIV-1 envelope. Vaccine 23 , 3836-3842 (2005).

95. Chionh, Y.T., Wee, J.L., Every, A.L., Ng, G.Z. \& Sutton, P. M-cell targeting of whole killed bacteria induces protective immunity against gastrointestinal pathogens. Infect. Immun. 77, 2962-2970 (2009).

96. Chionh, Y.T. \& Sutton, P. Targeting of whole killed bacteria to gastrointestinal $\mathrm{M}$-cells induces humoral immunity in the female reproductive tract. Gut Microbes 1, 42-44 (2010).

97. Gupta, P.N. \& Vyas, S.P. Investigation of lectinized liposomes as M-cell targeted carrier-adjuvant for mucosal administration. Colloids Surf. $B$ Biointerfaces 82, 118-125 (2011).

98. Misumi, S. et al. Targeted delivery of immunogen to primate $m$ cells with tetragalloyl lysine dendrimer. J. Immunol. 182, 6061-6070 (2009).

99. Rajapaksa, T.E., Stover-Hamer, M., Fernandez, X., Eckelhoefer, H.A. \& Lo, D.D. Claudin 4-targeted protein incorporated into PLGA nanoparticles can mediate M cell targeted delivery. J. Control. Release 142, 196-205 (2010).

100. Lo, D.D., Ling, J. \& Eckelhoefer, A.H. M cell targeting by a claudin 4 targeting peptide can enhance mucosal IgA responses. BMC Biotechnol. 12, 7 (2012)

101. Wu, Y. et al. M cell-targeted DNA vaccination. Proc. Natl. Acad. Sci. USA 98, 9318-9323 (2001).

102. Rynda, A. et al. Low-dose tolerance is mediated by the microfold cell ligand, reovirus protein sigma 1. J. Immunol. 180, 5187-5200 (2008).

103. Suzuki, H. et al. Ovalbumin-protein sigma $1 \mathrm{M}$-cell targeting facilitates oral tolerance with reduction of antigen-specific CD4 ${ }^{+}$T cells. Gastroenterology 135, 917-925 (2008).

104. Kujala, P. et al. Prion uptake in the gut: identification of the first uptake and replication sites. PloS Pathogens 7, e1002449 (2011).

105. Sasaki, I. et al. Spi-B is crucial for plasmacytoid dendritic cell function and development. Blood 120, 4733-4743 (2012).

106. Su, G.H. et al. Defective B cell receptor-mediated responses in mice lacking the Ets protein. EMBO J. 16, 7118-7129 (1997).

107. McDole, J.R. et al. Goblet cells deliver luminal antigen to $\mathrm{CD}_{103}{ }^{+}$ dendritic cells in the small intestine. Nature 483, 345-349 (2012).

108. Rescigno, M. et al. Dendritic cells express tight junction proteins and penetrate gut epithelial monolayers to sample bacteria. Nat. Immunol. 2, 361-367 (2001)

109. Farache, J. et al. Liminal bacteria recruit CD103 + dendritic cells into the intestinal epithelium to sample bacterial antigens for presentation. Immunity 38, 581-595 (2013).

110. Delamarre, L., Pack, M., Chang, H., Mellman, I. \& Trombetta, E.S. Differential lysosomal proteolysis in antigen-presenting cells determines antigen fate. Science 307, 1630-1634 (2005).

111. Bergtold, A., Desai, D.D., Gavhane, A. \& Clynes, R. Cell surface recycling of internalized antigen permits dendritic cell priming to B cells. Immunity 23, 503-514 (2005).

112. Wykes, M., Pombo, A., Jenkins, C. \& MacPherson, G.G. Dendritic cells interact directly with naive $B$ lymphocytes to transfer antigen and initiate class switching in a primary T-dependent response. J. Immunol. 161, 1313-1319 (1998).

113. Macpherson, A.J. \& Uhr, T. Induction of protective IgA by intestinal dendritic cells carrying commensal bacteria. Science 303, 1662-1665 (2004).

114. Huang, F.-P. et al. A discrete subpopulation of dendritic cells transports apoptotic intestinal epithelial cells to T cell areas of mesenteric lymph nodes. J. Exp. Med. 191, 435-443 (2000).

115. Lapthorne, S. et al. Differential intestinal M-cell gene expression response to gut commensals. Immunology 136, 312-324 (2012). 